\title{
The CHIANTI Database
}

\author{
E. Landi, K.P. Dere
}

Naval Research Laboratory, Washington, DC 20375, USA

P.R. Young

Rutherford Appleton Laboratory, Chilton, Didcot, OX11 OQX, UK

\section{Landini}

University of Florence, Largo E.Fermi 2, 51025 Firenze, Italy

\author{
H.E. Mason, G. Del Zanna
}

Dept. of Appl. Math. and Theor. Phys., University of Cambridge, UK

\begin{abstract}
The CHIANTI database contains assessed atomic energy levels, wavelengths, radiative transition probabilities and excitation rates necessary to calculate line emissivities and synthetic spectra of a large number of ions of astrophysical interest. CHIANTI also includes a suite of programs to carry out plasma diagnostics. In the present paper we describe the contents of the CHIANTI database, its main applications and its future developments.
\end{abstract}

\section{Introduction}

The launch of new space-born spectroscopic instrumentation has led to an increased need for a comprehensive and accurate database of atomic data that enables the astrophysical community to interpret the wealth of observed data now available. Over the years, we have built up the CHIANTI database in an effort to provide the astrophysical community with a database that would allow spectral analysis, plasma diagnostics and synthetic spectra calculations to be carried out at wavelengths from X-rays to the UV.

CHIANTI has been continually expanded since its first release in 1997 (Dere et al. 1997), and now includes new data for minor ions and continuum radiation (version 2 - Landi et al. 1999), data for the X-ray wavelength range (version 3 - Dere et al. 2001), and proton excitation rates and photoexcitation (version 4, Young et al. 2003). CHIANTI synthetic spectra have been compared with highresolution spectral observations of the Sun and have been found to be complete and accurate in most cases (Young et al. 1998, Landi et al. 2002a,b, Del Zanna et al. 2002).

CHIANTI has been used extensively by the solar physics community, and has become the standard resource for atomic data for the interpretation of the solar spectrum. Also, CHIANTI has been extensively used for the analysis of data from Hubble, EUVE, FUSE, XMM, and Chandra. The accuracy 
and completeness of the CHIANTI database has led to the inclusion of CHIANTI emissivities in the software of current (SOHO/EIT and TRACE) and future (STEREO/SECCHI, Solar-B/XRT) broad-band imagers observing the Sun. CHIANTI is also part of the data analysis software for the SOHO/CDS spectrometer, and will be used for the Solar-B/EIS spectrometer to be launched in 2006. Because of their completeness and accessibility, CHIANTI data have been included in other spectral codes: XSTAR (Bautista \& Kallman 2001), APEC/APED (Smith et al. 2001) and the Arcetri Code (Landi \& Landini 2002).

CHIANTI is freely distributed by SolarSoft and it is also available at the web site

$$
\text { http : //wwwsolar.nrl.navy.mil/chianti.html. }
$$

\section{CHIANTI data}

CHIANTI calculates line and continuum emission of optically thin plasmas, and includes the following processes:

- spectral line emission for more than 200 ions;

- radiative recombination continuum;

- bremsstrahlung continuum;

- Two-photon continuum.

Spectral line emission is calculated by solving the equations for level population under the assumption of statistical equilibrium, including electron and proton excitation, stimulated and spontaneous radiative decay and photoexcitation. All data come from the refereed literature.

The aim of the CHIANTI database is to provide a compact and easy-toaccess set of data that is completely transparent to the user. CHIANTI data are stored in $A S C I I$ files, where the original literature sources of the data are reported at the end of each file.

\section{Applications}

Plasma diagnostics: CHIANTI emissivities have been used to carry out plasma diagnostics on a variety of astrophysical objects to measure plasma density, temperature, elemental abundances, velocities, emission measure, and differential emission measure in many different sources.

For example, Feldman et al. (2001) found agreement between the observed spectrum of the Jupiter-Io plasma torus and CHIANTI predictions, and estimates of the electron density and temperature made by CHIANTI complement in-situ measurements made by the Voyager and Galileo spacecrafts.

CHIANTI data have been used to measure physical properties of the solar atmosphere, in a wide variety of conditions: coronal holes (Del Zanna \& Bromage 1999), quiet Sun (Landi et al. 2000), active regions (Mason et al. 1999), and flares (Landi et al. 2003). Plasma structures in the solar atmosphere, such as loops, have also been studied (i.e. Schmelz et al. 2001).

Density sensitive line pairs have been used to measure electron density in classical T-Tauri stars by Johns-Krull et al. (2000), using UV spectral lines 
observed with IUE and CHIANTI emissivities. Such estimates help in discriminating between different accretion shock models and allow the search for correlations between density and mass accretion rate.

CHIANTI has also been applied to X-ray and UV spectra of cool stars by a number of authors, in order to investigate the quiescent and active state of their coronae. For example, Robinson et al. (2001) measured plasma emission measure, temperature and density using CHIANTI and UV/optical observations from Hubble. Woitke \& Sedlmayr (1999) used CHIANTI data for Fe II to investigate the role of iron in the energy balance in the extended atmospheres and circum-stellar envelopes of cool stars. Swartz et al. (2002) used CHIANTI to model the X-ray emission from sources detected by Chandra in M81.

Dixon et al. (2001) used O VI emissivities from CHIANTI to address the problem of the excess of EUV and soft X-ray flux present in the spectra of two clusters of galaxies, concluding that current warm gas models of the EUV excess should be revised.

Instrument calibration: In order to determine quantitative information from broad-band imagers, such as SOHO/EIT and TRACE, it is necessary to understand precisely the spectrum that is detected in each of the instruments' band passes. CHIANTI has been adopted as part of the standard EIT and TRACE calibration software to convert count rates into temperature and emission measure maps.

Comparison of line intensity ratios predicted by CHIANTI and observations from high-resolution spectrometers can provide a powerful means for determining their relative intensity calibration. Such technique has been recently applied to the SERTS rocket-borne spectrometer by Brosius et al. (1998) and Young et al. (1998). Del Zanna et al. (2001) applied CHIANTI to the calibration of $\mathrm{SOHO} / \mathrm{CDS}$, determining the relative intensity calibration of its six channels.

Atomic data assessment: The accuracy of atomic data and transition rates is crucial to reliable plasma diagnostics. The accuracy can only be assessed by comparison with observations, but direct laboratory measurements of transition rates are difficult. Comparison between CHIANTI predictions and line ratios observed by high-resolution spectrometers can be a powerful tool to investigate the accuracy of transition probabilities.

As part of the CHIANTI project we have compared CHIANTI emissivities with observations from the 1989 SERTS flight (Young et al. 1998), SUMER and CDS (Landi et al. 2002a,b). The comparisons have shown excellent agreement for the vast majority of ions, and CHIANTI accounts for all the most important lines in the EUV spectral range. Also a few ions whose atomic data need improvements were identified in these works (i.e. Fe XII). These comparisons have triggered new calculations aimed at improving the data available in CHIANTI and in the literature.

Of particular importance is the comparison between CHIANTI and the $\mathrm{X}$-ray spectra observed with Chandra and XMM. Preliminary work has been carried out by Del Zanna et al. (2002), who showed that CHIANTI is able to reproduce most of the observed lines, but in the 10 to $13 \AA$ wavelength range more work is needed to include the $n \geq 4$ transitions of highly ionized iron ions. 


\section{Future steps}

The CHIANTI team is committed to continually update and expand the CHIANTI database in order to include more accurate data and more processes. The two main areas of development currently underway in CHIANTI are

1: Inclusion of ionization and recombination rates

2: Inclusion of data for the $n \geq 4$ transitions

Ionization and recombination rates will be included to enable CHIANTI users to investigate the effects of transient conditions often present in astrophysical plasmas. The inclusion of data for the $n \geq 4$ transitions will improve dramatically the emissivities in the $\mathrm{X}$-ray range.

Acknowledgments. E. Landi is supported by NASA. K.P. Dere is supported by the Office of Naval Research. G. Del Zanna and H.E. Mason are supported by PPARC.

\section{References}

Bautista, M.A., Kallman, T.R., 2001, ApJS, 134, 139

Brosius, J.W., Davila, J.M., Thomas, R.J., 1998, ApJL, 497, L113

Del Zanna, G., Bromage, B.I.J., 1999, J. Geoph. Res., 104, 9753

Del Zanna, G., et al., 2001, A\&A, 379, 708

Del Zanna, G., et al., 2002, X-Ray Astronomy 2000: PASP Conf. Ser. 234, Giacconi, Stella \& Serio Eds.

Dere, K.P., et al., 1997, A\&AS 125,149

Dere, K.P., et al., 2001, ApJS, 134, 331

Dixon, W., et al., 2001, ApJ, 550, L25

Feldman, P.D., et al., 2001, ApJ 554, L123

Johns-Krull, C.M., Valenti, J.A., Linsky, J.L., 2000, ApJ, 539, 815

Landi, E., et al., 1999, A\&ASS, 135, 171

Landi, E., Mason, H.E., Lemaire, P., Landini, M., 2000, A\&A, 357, 743

Landi, E., Landini, M., 2002, A\&A, 384, 1124

Landi, E., Feldman, U., Dere, K.P., 2002a, ApJS, 139, 281

Landi, E., Feldman, U., Dere, K.P., 2002b, ApJ, 574, 495

Landi, E., et al., 2003, ApJ, 582, 506

Mason, H.E., Landi, E., Pike, C.D., Young, P.R., 1999, Sol.Phys., 189,129

Robinson, R.D., et al., 2001, ApJ, 554, 368

Schmelz, J.T., et al., 2001, ApJ, 556, 896

Smith, R.K., et al., 2001, ApJ, 556, 91

Swartz, D.A. et al., 2002, ApJ, 574, 382

Young, P.R., Landi, E., Thomas, R.J., 1998, A\&A, 329, 291

Young, P.R., et al., 2003, ApJS, 144, 135

Woitke, P., Sedlmayr, E., 1999, A\&A, 347, 617 